\title{
Characterization of the p-conformally flat \\ Riemannian manifold
}

\author{
By Izumi Hasegawa
}

(Received May 14, 1975)

\section{§1. Introduction.}

Recently, Bang-yen Chen and Kentaro Yano [1] proved the following:

THEOREM 1. In order that a Riemannian manifold $M$ of dimension $n>3$ is conformally flat, it is necessary and sufficient that there exists a (unique) quadratic form $Q$ on $M$ such that the sectional curvature $K(\sigma)$ with respect to a plane $\sigma$ is the trace of the restriction of $Q$ to $\sigma$, i.e. $K(\sigma)=$ trace $Q / \sigma$, the metric being also restricted to $\sigma$.

The object of this paper is to give the generalization of this theorem, ipso facto, the characterization of higher order conformally flatness.

We have the following:

THEOREM 2. In order that a Riemannian manifold $M$ of dimension $n \geqq 4 p$ is p-conformally flat, it is necessary and sufficient that there exists $a$ (unique) quadratic form $Q$, which satisfies the generalized first Bianchi identity as double form of type $(2 p-1,2 p-1)$, on the bundle $\Lambda^{2 p-1}(M)$ of $(2 p-1)$-vectors of $M$ such that the $2 p$-th sectional curvature $K_{2 p}(\boldsymbol{\sigma})$ with respect to an $2 p$-plane $\sigma$ is the trace of the restriction of $Q$ to $\Lambda^{2 p-1}(\sigma)$, i.e. $K_{2 p}(\boldsymbol{\sigma})=$ trace $Q / \Lambda^{2 p-1}(\boldsymbol{\sigma})$.

\section{§2. Preliminaries.}

Let $M$ be an $n$-dimensional Riemannian manifold with the Riemannian metric $g$, let $\mathfrak{F}(M)$ be the algebra of functions on $M$ and let $\mathfrak{X}(M)$ be the Lie algebra of vector fields on $M$. In what follows we write $g=\langle$,$\rangle ,$ where it is convenient.

For $p$ an integer between 1 and $n$, let $\Lambda^{p}(M)$ denote the bundle of $p$ vectors of $M$ and let $\Lambda^{p}(m)$ be the fiber over $m \in M . \Lambda^{p}(M)$ is a Riemannian vector bundle, with the inner product on the fiber $\Lambda^{p}(m)$ over $m$ related to the inner product on the tangent space $M_{m}$ of $M$ at $m$ by

$$
\left\langle X_{1} \wedge X_{2} \wedge \cdots \wedge X_{p}, Y_{1} \wedge Y_{2} \wedge \cdots \wedge Y_{p}\right\rangle=\operatorname{det}\left[\left\langle X_{i}, Y_{j}\right\rangle\right],\left(X_{i}, Y_{j} \in M_{m}\right) .
$$

We define a double form of type $(p, q)$ on $M$ to be an $\mathfrak{F}(M)$-multilinear map 


$$
\omega: \mathfrak{X}(M)^{p} \times \mathfrak{F}(M)^{q} \longrightarrow \mathfrak{X}(M),
$$

which is skew-symmetric in the first $p$-variables and also in the last $q$ variables. We shall use the notation

$$
\omega\left(X_{1}, \cdots, X_{p}\right)\left(Y_{1}, \cdots, Y_{q}\right)
$$

to denote the value of $\omega$ on the vector fields $X_{1}, \cdots, X_{p}, Y_{1}, \cdots, Y_{q} \in \mathfrak{X}(M)$. If $p=q$ and

$$
\omega\left(X_{1}, \cdots, X_{p}\right)\left(Y_{1}, \cdots, Y_{p}\right)=\omega\left(Y_{1}, \cdots, Y_{p}\right)\left(X_{1}, \cdots, X_{p}\right),
$$

we say that $\omega$ is symmetric.

Let $\omega$ be a symmetric double form of type $(p, p)$. Hence, at each point $m \in M$, we may regard $\omega$ as aquadratic form on $\Lambda^{p}(m)$, i. e.

$$
\omega\left(X_{1}, \cdots, X_{p}\right)\left(Y_{1}, \cdots, Y_{p}\right)=\omega\left(X_{1} \wedge \cdots \wedge X_{p}, Y_{1} \wedge \cdots \wedge Y_{p}\right),\left(X_{i}, Y_{j} \in M_{m}\right) .
$$

Next, let $\omega$ be a double form of type $(p, q)$ and let $\theta$ be of type $(r, s)$ respectively. The exterior product $\omega \wedge \theta$ of $\omega$ and $\theta$ is defined by the formula

$$
\begin{aligned}
& (\omega \wedge \theta)\left(X_{1}, \cdots, X_{p+r}\right)\left(Y_{1}, \cdots, Y_{q+s}\right) \\
& \quad=\sum_{\substack{o \in g h(p, r) \\
\tau \in \varepsilon h(q), s)}} \mathcal{E}_{\sigma} \mathcal{E}_{\tau} \omega\left(X_{o(1)}, \cdots, X_{o(p)}\right)\left(Y_{\tau(1)}, \cdots, Y_{\tau(q)}\right) \\
& \quad \times \theta\left(X_{\sigma(p+1)}, \cdots, X_{\sigma(p+r)}\right)\left(Y_{\tau(q+1)}, \cdots, Y_{\tau(q+s)}\right), \quad\left(X_{i}, Y_{j} \in \mathfrak{X}(M)\right) .
\end{aligned}
$$

Here, $S h(p, r)$ denotes the set of all $(p, r)$-shuffles; specifically

$$
S h(p, r)=\left\{\sigma \in S_{p+r} \mid \sigma(1)<\cdots<\sigma(p) \text { and } \sigma(p+1)<\cdots<\sigma(p+r)\right\},
$$

where $S_{p+r}$ is the symmetric group of degree $p+r$.

It is easy to show that $\Lambda$ is an associative multiplication and

$$
\omega \wedge \theta=(-1)^{p r+q s} \theta \wedge \omega
$$

where $\omega$ has type $(p, q)$ and $\theta$ has type $(r, s)$.

Let $\omega^{k}$ denote the $k$-th exterior power of $\omega$. Then we can rewrite the inner product on $\Lambda^{p}(m)$ over $m \in M$ as follows ;

$$
\left\langle X_{1} \wedge \cdots \wedge X_{p}, Y_{1} \wedge \cdots \wedge Y_{p}\right\rangle=\frac{1}{p !} g^{p}\left(X_{1}, \cdots, X_{p}\right)\left(Y_{1}, \cdots, Y_{p}\right) .
$$

We define the double form $c \omega$ of type $(p-1, q-1)$ for $\omega$ of type $(p$, $q$ ) as follows. With $p=0$ or $q=0$, we put $c \omega=0$. If both $p$ and $q \geqq 1$, then we put

$$
\begin{aligned}
& c \omega\left(X_{1}, \cdots, X_{p-1}\right)\left(Y_{1}, \cdots, Y_{q-1}\right) \\
& \quad=\sum_{k=1}^{n} \omega\left(E_{k}, X_{1}, \cdots, X_{p-1}\right)\left(E_{k}, Y_{1}, \cdots, Y_{q-1}\right),
\end{aligned}
$$


where $\left\{E_{1}, \cdots, E_{n}\right\}$ is a locally defined orthonormal frame field with respect to $g$. We call this map $c$ the contraction.

The double form $b \omega$ of type $(p+1, q-1)$ for $\omega$ of type $(p, q)$ is defined as follows. With $q=0$, we put $b \omega=0$. If $q \geqq 1$, then we put

$$
\begin{aligned}
& b \omega\left(X_{1}, \cdots, X_{p+1}\right)\left(Y_{1}, \cdots, Y_{q-1}\right) \\
& \quad=\sum_{j=1}^{p+1}(-1)^{j} \omega\left(X_{1}, \cdots, \check{X}_{j}, \cdots, X_{p+1}\right)\left(X_{j}, Y_{1}, \cdots, Y_{q-1}\right),
\end{aligned}
$$

where the symbol $`$ denotes omission.

Note that $b \omega=0$ for any quadratic form $\omega$ on $M$. Of course, we have $b g=0$. If $b \omega=0$, we call $\omega$ a double form satisfying the generalized first Bianchi identity. We know that the double form of type $(p, p)$ satisfying the generalized first Bianchi identity is symmetric.

Concerning these operators, the following lemmas are well known (cf. see [2] and [3]).

Lemma 1. Let $\omega$ and $\theta$ be the double forms on $M$ of types $(p, q)$ and $(r, s)$ respectively. Then we have the following formulas:

$$
\begin{aligned}
& c(g \wedge \omega)=g \wedge c \omega+(n-p-q) \omega, \\
& b(\omega \wedge \theta)=b \omega \wedge \theta+(-1)^{p+q} \omega \wedge b \theta, \\
& b c=c b .
\end{aligned}
$$

Lemma 2. Let $\omega$ be a double form of type $(p, p)$ on $M$. Suppose that $b \omega=0$ and $\omega\left(X_{1}, \cdots, X_{p}\right)\left(X_{1}, \cdots, X_{p}\right)=0$ for all $X_{1}, \cdots, X_{p} \in M_{m}$ at each point $m \in M$. Then $\omega=0$.

Let $R_{X Y}$ be the curvature operator given by the formula:

$$
R_{X Y}=\left[\nabla_{X}, \nabla_{Y}\right]-\nabla_{[X, Y]}, \quad(X, Y \in \mathfrak{X}(M)) .
$$

Then we define a curvature double form $R$ of type $(2,2)$ by the formula:

$$
R(X, Y)(Z, W)=\left\langle R_{X Y} Z, W\right\rangle, \quad(X, Y, Z, W \in \mathfrak{X}(M)) .
$$

Note that $b R=0$ reduces to the first Bianchi identity.

The Weyl conformal curvature tensor $C$ is a double form of type $(2,2)$ is given by

$$
C=R-\frac{1}{n-2} g \wedge c R+\frac{c^{2} R}{2(n-2)(n-1)} g^{2} .
$$

As the generalization of the conformal curvature tensor $C$, we define the $p$-th conformal curvature tensor $C_{p}$ by the formula [2]:

$$
C_{p}=R^{p}+\sum_{k=1}^{2 p} \frac{(-1)^{k}}{k ! \Pi_{j=0}^{k-1}(n-4 p+2+j)} g^{k} \wedge c^{k} R^{p}, \quad(n \geqq 4 p-1) .
$$


The manifold $M$ is called $p$-conformally flat if $n>4 p-1$ and $C_{p}=0$. Of course, if $p=1$, then $M$ is conformally fiat.

Let $G_{2 p}(M)$ be a Grassman bundle of oriented tangent $2 p$-planes of $M$. The $2 p$-th sectional curvature $K_{2 p}(\sigma)$ with respect to $\sigma=(m, P) \in G_{2 p}(M)$ is given by the formula [3]:

$$
K_{2 p}(\sigma)=\frac{(-2)^{p} R^{p}\left(X_{1}, \cdots, X_{2 p}\right)\left(X_{1}, \cdots, X_{2 p}\right)}{(2 p) !\left\|X_{1} \wedge \cdots \wedge X_{2 p}\right\|^{2}}
$$

where $P$ is spanned by $X_{1}, \cdots, X_{2 p} \in M_{m}$.

\section{§3. Proof of Theorem 2 .}

Suppose that $M$ is a $p$-conformally flat Riemannian manifold of dimension $n \geqq 4 \mathrm{p}$. Then we have

$$
C_{p}=0 \text {, }
$$

that is

$$
R^{p}=\sum_{k=1}^{2 p} \frac{(-1)^{k-1}}{k ! \Pi_{j=0}^{k-1}(n-4 p+2+j)} g^{k} \wedge c^{k} R^{p} .
$$

The $2 p$-th sectional curvature $K_{2 p}(\sigma)$ with respect to $2 p$-plane $\sigma=(m, P) \epsilon$ $G_{2 p}(M)$ is given by

$$
\begin{aligned}
K_{2 p}(\boldsymbol{\sigma})= & \frac{1}{\left\|X_{1} \wedge \cdots \wedge X_{2 p}\right\|^{2}} \\
& \times\left\{\sum_{i, j=1}^{2 p} g\left(X_{i}, X_{j}\right) Q\left(X_{1}, \cdots, \check{X}_{i}, \cdots, X_{2 p}\right)\left(X_{1}, \cdots, \check{X}_{j}, \cdots, X_{2 p}\right)\right\}
\end{aligned}
$$

where $Q=\frac{(-2)^{p}}{(2 p) !} \sum_{k=1}^{2 p} \frac{(-1)^{k-1}}{k ! \Pi_{j=0}^{k-1}(n-4 p+2+j)} g^{k-1} \wedge c^{k} R^{p}$

and $P$ is spanned by $X_{1}, \cdots, X_{2 p} \in M_{m}$.

Thus if $\left\{X_{1}, \cdots, X_{2 p}\right\}$ is any orthonormal basis for $P$, then we obtain

$$
K_{2 p}(\sigma)=\sum_{j=1}^{2 p} Q\left(X_{1} \wedge \cdots \wedge \check{X}_{j} \wedge \cdots \wedge X_{2 p}\right)\left(X_{1} \wedge \cdots \wedge \check{X}_{j} \wedge \cdots \wedge X_{2 p}\right),
$$

that is, the $2 p$-th sectional curvature $K_{2 p}(\sigma)$ with respect to $\sigma$ is given by the trace of the restriction of $Q$ to $\sigma$.

From Lemma 1, the generalized first Bianchi identity $b Q=0$ for $Q$ is straightforward.

Conversely, suppose that the $2 p$-th sectional curvature $K_{2 p}(\sigma)$ with respect to $\sigma=(m, P) \in G_{2 p}(M)$ is given by

$$
K_{2 p}(\sigma)=\text { trace } Q / \Lambda^{2 p-1}(\sigma),
$$


where $Q$ is a certain quadratic form on $\Lambda^{2 p-1}(m)$ which satisfies the generalized first Bianchi identity as a double form of type $(2 p-1,2 p-1)$. The expression trace $Q / \Lambda^{2 p-1}(\sigma)$ being independent of the choice of the orthonormal basis $\left\{U_{1}, \cdots, U_{2 p}\right\}$ of $A^{2 p-1}(m)$ respected to $P$, we put

$$
\begin{aligned}
U_{1} & =\frac{\widetilde{X}_{1}}{\left\|\widetilde{X}_{1}\right\|}, \\
U_{2} & =\frac{\left\|\widetilde{X}_{1}\right\|^{2} \widetilde{X}_{2}-\left\langle\widetilde{X}_{1}, \widetilde{X}_{2}\right\rangle \widetilde{X}_{1}}{\left\|\widetilde{X}_{1}\right\|\left\|\widetilde{X}_{1} \wedge \widetilde{X}_{2}\right\|}, \\
\vdots & \sum_{k=1}^{2 p}(-1)^{k}\left\langle\widetilde{X}_{1} \wedge \cdots \wedge \widetilde{X}_{2 p-1}, \widetilde{X}_{1} \wedge \cdots \wedge \widetilde{X}_{k} \wedge \cdots \wedge \widetilde{X}_{2 p}\right\rangle \widetilde{X}_{k} \\
U_{2 p} & \left\|\widetilde{X}_{1} \wedge \cdots \wedge \widetilde{X}_{2 p-1}\right\|\left\|\widetilde{X}_{1} \wedge \cdots \wedge \widetilde{X}_{2 p}\right\|
\end{aligned}
$$

where $\widetilde{X}_{i}=X_{1} \wedge \cdots \wedge \breve{X}_{i} \wedge \cdots \wedge X_{2 p} \in \Lambda^{2 p-1}(m)$ and $\wedge$ is an exterior multiplication on $\Lambda^{2 p-1}(m)$.

Then from (3.5) and (3.6), we have

$$
\begin{aligned}
& \frac{(-2)^{p} R^{p}\left(X_{1}, \cdots, X_{2 p}\right)\left(X_{1}, \cdots, X_{2 p}\right)}{(2 p) !\left\|X_{1} \wedge \cdots \wedge X_{2 p}\right\|^{2}} \\
& =\frac{\sum_{i, j=1}^{2 p}(-1)^{i+j}\left\langle\widetilde{X}_{1} \wedge \cdots \wedge \widetilde{X}_{i} \wedge \cdots \wedge \widetilde{X}_{2 p}, \widetilde{X}_{1} \wedge \cdots \wedge \widetilde{X}_{j} \wedge \cdots \wedge \widetilde{X}_{2 p}\right\rangle Q\left(\widetilde{X}_{i}, \widetilde{X}_{j}\right)}{\left\|\widetilde{X}_{1} \wedge \cdots \wedge \widetilde{X}_{2 p}\right\|^{2}}
\end{aligned}
$$

Here we use the following identities;

$$
\begin{aligned}
& \left\|\widetilde{X}_{1} \wedge \cdots \wedge \widetilde{X}_{2 p}\right\|^{2}=\left\|X_{1} \wedge \cdots \wedge X_{2 p}\right\|^{4 p-2} \\
& \quad\left\langle\widetilde{X}_{1} \wedge \cdots \wedge \widetilde{X}_{i} \wedge \cdots \wedge \widetilde{X}_{2 p}, \widetilde{X}_{1} \wedge \cdots \wedge \widetilde{X}_{j} \wedge \cdots \wedge \widetilde{X}_{2 p}\right\rangle \\
& \quad=\left\|X_{1} \wedge \cdots \wedge X_{2 p}\right\|^{4 p-4}\left\langle X_{i}, X_{j}\right\rangle
\end{aligned}
$$

Using this fact, we find

$$
\begin{aligned}
& \frac{(-2)^{p}}{(2 p) !} R^{p}\left(X_{1}, \cdots, X_{2 p}\right)\left(X_{1}, \cdots, X_{2 p}\right) \\
& \quad=(g \wedge Q)\left(X_{1}, \cdots, X_{2 p}\right)\left(X_{1}, \cdots, X_{2 p}\right) .
\end{aligned}
$$

From Lemm 2, we obtain

$$
R^{p}=g \wedge L
$$

where $L=\frac{(2 p) !}{(-2)^{p}} Q$.

From (3.10) and Lemma 1, we have 
(3.11)

$$
\begin{aligned}
& c R=g \wedge c L+(n-4 p+2) L, \\
& \vdots \\
& c^{k} R^{p}=g \wedge c^{k} L+k(n-4 p+k+1) c^{k-1} L,
\end{aligned}
$$$$
c^{2 p} R^{p}=2 p(n-2 p+1) c^{2 p-1} L \text {. }
$$

Then it follows that

$$
L=\sum_{k=1}^{2 p} \frac{(-1)^{k-1}}{k ! \Pi_{j=0}^{k-1}(n-4 p+2+j)} g^{k-1} \wedge c^{k} R^{p}, \quad \text { i.e. } \quad \text { (3.2). }
$$

Therefore $M$ is $p$-conformally flat.

\section{References}

[1] B.-Y. CHEN and K. YANO: Manifolds with vanishing Weyl or Bochner curvature tensor, J. Math. Soc. Japan, 27 (1975), 106-112.

[2] R. S. Kulkarni: On the Bianchi Identities, Math. Ann., 199 (1972), 175-204.

[3] A. Gray: Some Relations between Curvature and Characteristic Classes, Math. Ann., 184 (1970), 257-267.

[4] J. A. ThORPE: Sectional curvatures and Characteristic Classes, Ann. of Math., 80 (1964), 429-443.

[5] T. NASU: On Conformal Invariants of Higher Order, Hiroshima Math. J., 5 (1975), 43-60. 